INPLASY

PROTOCOL

To cite: Samsudin et al. Law enforcement and preparedness for airborne and droplet borne infectious disease in industries: A systematic review. Inplasy protocol 202190049. doi: 10.37766/inplasy2021.9.0049

Received: 16 September 2021

Published: 16 September 2021

Corresponding author:

Siti Munira Yasin

smunira@uitm.edu.my

Author Affiliation:

Universiti Teknologi MARA

(UiTM), Department of

Occupational Health and

Safety (DOSH).

Support: Grant Provided by DOSH.

Review Stage at time of this submission: Formal screening of search results against eligibility criteria.

Conflicts of interest:

None declared.

\section{Law enforcement and preparedness for airborne and droplet borne infectious diseases in industries: $A$ systematic review}

Samsudin, EZ1; Yasin, SM2; Ismail, N3; Isa, MR4; Rahman, NA5; Abdullah Hair, AF6; Manivasagam, D7; Rosli, NFA8.

Review question / Objective: A structured, systematic review and qualitative synthesis of peer-reviewed publications will perform to explore law enforcement and preparedness for airborne and droplet borne infectious diseases in industries. Information sources: We will be using three biomedical electronic databases (Pubmed, Scopus, and Web of Science), one economic database (IDEAS/REPEC) and three occupational safety and health databases (OSHLINE, HSELINE, and NIOSHTIC-2).

INPLASY registration number: This protocol was registered with the International Platform of Registered Systematic Review and Meta-Analysis Protocols (INPLASY) on 16 September 2021 and was last updated on 16 September 2021 (registration number INPLASY202190049).

\section{INTRODUCTION}

Review question / Objective: A structured, systematic review and qualitative synthesis of peer-reviewed publications will perform to explore law enforcement and preparedness for airborne and droplet borne infectious diseases in industries.

Condition being studied: Law enforcement and preparedness for airborne and droplet borne infectious diseases in industries. After searches have been performed, 
articles will then be organized into EndNote 20 Software and duplicates were identified and removed. This will be performed by one reviewer, via the "Find and Remove Duplicate References" function at first, which will be followed by manual screening, as some of the same articles were entered slightly differently into different databases. After duplicates have been removed, articles were assessed for eligibility independently by two reviewers in two stages. In stage one, the title and abstract of search results will be screened and assessed for relevance. In stage two, the full text of potentially relevant publications will be retrieved and reviewed for inclusion. Any primary study in English examining law enforcement and preparedness for airborne and droplet borne infectious diseases in any industry will be included. Studies that were nonhuman studies, reviews, editorials, commentaries, forewords, opinion pieces, and books, studies that examined infectious diseases transmitted via routes other than airborne and droplet borne transmission, as well as studies examining variables others law enforcement and preparedness were will be excluded. The reason for excluding a publication following title and abstract review as well as the fulltext review will be noted. Both stages of the study selection will be performed independently by two reviewers and crossvalidated to assess for disagreements. The list of studies that will be included and excluded based on the inclusion and exclusion criteria described earlier was cross-validated to assess for disagreements. For any disagreement that will present, consensus will be sought where possible, and in cases where that will not be possible, a third reviewer will be assigned. The per cent agreement and Cohen's Kappa for the study selection process were $X \%$ and $X$, respectively. Attempts to contact authors for articles that will not be available in the full text were made, and only full-text articles will be included in the review to enable quality assessments. Hand searching will not be attempted due to resource limitations.
Search strategy: A comprehensive search of the literature was undertaken in August 2021 using the electronic databases PubMed, Scopus, Web of Science, and Cochrane Library. The search aimed to identify relevant articles published in peerreviewed journals written in English, with the assumption that most of the important findings will be reported in English regardless of country of origin. The boolean search was performed on each database using the search terms: ("law enforcement" OR "legal enforcement" OR "regulatory enforcement" OR "standard enforcement" OR "policy enforcement" OR policing OR "control policy" OR "law preparedness" OR "legal preparedness" OR "legal readiness" OR "legal preparation" OR "preparedness policy" OR "preparedness plan" OR "emergency response plan" OR "disaster response plan" OR "preparedness strateg*") AND (infection OR "infectious disease" OR "communicable disease" OR outbreak OR epidemic OR pandemic OR COVID-19 OR SARS OR MERS OR coronavirus OR influenza OR adenovirus OR enterovirus OR rotavirus OR measles OR mumps OR smallpox OR tuberculosis OR diphtheria OR anthrax OR legionellosis OR meningococcus OR pneumococcus OR Bordetella OR aspergillosis OR blastomycosis OR cryptococcosis) AND (industr* OR workplace OR organization OR organisation OR compan*) The terms included in the Boolean search will be chosen after careful consideration of terms identified from the literature review. The Boolean search operator "OR" will be used to broaden the search with multiple analogous terms, while "AND" will be used to narrow the search to studies examining socioeconomic and occupational safety and the health impact of infectious disease and outbreaks in industries. The search will be conducted by one reviewer and will be performed to include title and abstract (PubMed), titles, abstract and keywords (Scopus), abstract (Web of Science), and title, abstract and keyword (Cochrane Library), without restriction to date or publication. All searches will be concluded by $X$. 
Participant or population: Workers.

Intervention: Not applicable.

Comparator: Not applicable.

Study designs to be included: The methodological quality of the included studies will be assessed by examining the level of evidence according to the Table of Evidence Levels from Cincinnati Children's Hospital Medical Center (CCHMC) (2012) and quality of study according to the Newcastle-Ottawa Scale (NOS).We will include a studies that will be assessed by examining the level of evidence.

Information sources: We will be using three biomedical electronic databases (Pubmed, Scopus, and Web of Science), one economic database (IDEAS/REPEC) and three occupational safety and health databases (OSHLINE, HSELINE, and NIOSHTIC-2).

Main outcome(s): A structured, systematic review and qualitative synthesis of peerreviewed publications will perform to explore law enforcement and preparedness for airborne and droplet borne infectious diseases in industries.

Quality assessment / Risk of bias analysis: Quality assessment will be referred to according to the NOS, which denotes good quality study and lesser quality study respectively in terms of methodological quality. The NOS is a widely used and validated tool developed by Wells et al. (2000) and incorporates the assessment of three broad perspectives for cohort and case-control studies: the selection of the study groups, the comparability of the groups, and the ascertainment of exposure or outcome of interest. The NOS has also been adapted for use in cross-sectional studies by Herzog et al. (2013), which was further adapted for this study in two areas: 1) For the ascertainment of the outcome, we have assigned two stars for validated measurement tool and/or organisational records, one star for a non-validated measurement tool that is based literature review or previous studies and/or self- report data, and no star for no description of the measurement tool, as our study was concerned with enforcement and preparedness which rely on study instruments and records rather than clinical outcome assessment, and 2) Included ascertainment of exposure under "Exposure, outcome and analysis" instead of "Selection". Based on a 'star system', a star was awarded for every quality criterion met by the study and the quality rating was assigned as follows: a) Cohort studies: 13 maximum stars and a final rating of $0-3$ stars as "poor", 4-6 stars as "moderate", 7-9 stars as "good" and 10-13 stars as "excellent" b) Case-control studies: 10 maximum stars and a final rating of 0-3 stars as "poor", 4-5 stars as "moderate", 6-7 stars as "good", and 8-10 stars as "excellent" c) Cross-sectional studies: 10 maximum stars and a final rating of $0-3$ stars as "poor", 4-5 stars as "moderate", 6-7 stars as "good", and 8-10 stars as "excellent". In the final quality rating, studies under the categories "excellent" and "good" will be rated as "a" and those under the categories "poor" and "moderate" will be rated as "b". The quality assessment will be performed independently by two reviewers. Data extraction and analysis will be crossvalidated to assess for disagreements. For any disagreement that will present, consensus will be sought where possible. In cases where that will not be possible, a third reviewer will be assigned.

Strategy of data synthesis: For each of the included study, data on the author, year of publication, study design, type of infectious disease, study population, location of study, number of participants that will be included, study instruments that will be use, study variables will be examine, socioeconomic impact, occupational and safety health impact, and study conclusion will be extracted. The data extraction will be performed independently by two reviewers. For any disagreement that was present, consensus will not seek where possible, and in cases where that will be not possible, a third reviewer will then be assign. Data will then analyse qualitatively due to the heterogeneity of studies that will 
be included in the systematic review, and meta-analysis will not attempt. Where applicable, data will not analyse using descriptive statistics using Statistical Package of Social Science Version 27. The numerical data will be analysed using mean and standard deviation, while the categorical data will be analysed using frequency and percentage.

Subgroup analysis: Not applicable.

Sensitivity analysis: Not applicable.

Language: English.

Country(ies) involved: Malaysia.

Keywords: law; enforcement; legal; occupational; safety; health; impacts; infectious; disease; workplaces.

Contributions of each author:

Author 1 - Ely Zarina Samsudin.

Email: elyzarina@uitm.edu.my

Author 2 - Siti Munira Yasin.

Email: smunira@uitm.edu.my

Author 3 - Nurhuda Ismail.

Email: yuda@uitm.edu.my

Author 4 - Muhammad Rodi Isa.

Email: rodi@uitm.edu.my

Author 5 - Nasaruddin Abd Rahman.

Email: nasa044@uitm.edu.my

Author 6 - Ahmad Fitri Abdullah Hair.

Email: ahmadfitri@mohr.gov.my

Author 7 - Dayanath A/L Manivasagam.

Email: dayanath@mohr.gov.my

Author 8 - Nur Fateh Alia Rosli.

Email: nurfatehalia97@gmail.com 